\title{
TOMOGRAFÍA DE COHERENCIA ÓPTICA EN LA DISTROFIA MACULAR VITELIFORME TIPO 2
}

\section{OPTICAL COHERENCE TOMOGRAPHY IN VITELLIFORM MACULAR DYSTROPHY TYPE 2}

\author{
VILLENA-IRIGOYEN O ${ }^{1}$, ESPEJO-DE-LOS-RISCOS E ${ }^{1}$, ESPAÑA-CONTRERAS M ${ }^{1}$, \\ JÓDAR-MÁRQUEZ M${ }^{1}$, MAÑAS-UXO C ${ }^{1}$
}

\section{RESUMEN}

Caso clínico: Presentamos el caso de un niño de 10 años con una distrofia macular viteliforme bilateral, en progresión, y el padre con estadios finales en ambos ojos; se estudia la evolución con la tomografía óptica de coherencia.

Discusión: La tomografía óptica de coherencia es una herramienta útil y no invasiva, que permite complementar el diagnóstico y el seguimiento de los pacientes, aportando datos morfológicos de la lesión así como cambios secundarios en la retina adyacente. Demuestra la localización del material amarillento depositado bajo la retina sensorial.

Palabras clave: Distrofia macular viteliforme, Distrofia Vitelina, Degeneración Viteloeruptiva, Enfermedad de Best, Tomografía de coherencia óptica.

\begin{abstract}
Case report: We report the case of a 10-year-old boy with a progressive bilateral vitelliform macular dystrophy, and his father with terminal stage disease in both eyes; we studied the development and progression of this condition with optical coherence tomography.

Discussion: Optical coherence tomography is a useful noninvasive tool that complements other diagnostic modalities and improves the follow up assessment. It provides additional information on the morphology of the lesion as well as identifying secondary changes in the adjacent retina. It also demonstrates the location of any yellowish material under the sensory retina (Arch Soc Esp Oftalmol 2008; 83: 501-504).
\end{abstract}

Key words: Vitelliform macular dystrophy, Vitelline dystrophy, Vitelliruptive degeneration, Best's disease, Optical coherence tomography.

\section{INTRODUCCIÓN}

La enfermedad de Best o distrofia viteliforme tipo 2 (VMD 2), es una distrofia del epitelio pigmentario retiniano (EPR). Se engloba entre las distrofias maculares hereditarias (autosómica domi- nante). El gen implicado en la enfermedad de Best está en el cromosoma 11q12.3 que codifica la bestrofina (1). Se produce un depósito variable de material amarillento a menudo atribuido al depósito lipofucsina en el epitelio pigmentario retiniano (EPR) (1).

\footnotetext{
Recibido: 5/2/07. Aceptado: 23/6/08.

Hospital Regional Carlos Haya. Servicio de Oftalmología. Málaga. España.

1 Licenciado en Medicina.

Caso 1 presentado parcialmente en atlas de OCT (autores: Dra. España y cols.).

Correspondencia:

Ólga Villena Irigoyen

Hospital Regional Carlos Haya

C/. Cervantes, $5,4 .^{\circ} \mathrm{D}$

29016 Málaga

España

E-mail: olgavillena@yahoo.es
} 
En niños de 3 a 15 años se puede ver la evolución de la distrofia viteliforme con los siguientes estadios: fóvea normal (con alteración del electrooculograma - EOG_-) o estadio previteliforme, estadio viteliforme, estadio de pseudohipopión, en yema batida y estadio atrófico- cicatricial.

\section{CASOS CLÍNICOS}

\section{Caso 1}

Presentamos el caso de un niño de 10 años que se diagnostica de forma casual en consulta de una maculopatía viteliforme bilateral. Cabe destacar el antecedente familiar paterno de la enfermedad de Best (autosómico dominante) en estadio final.

La agudeza visual (AV) que presenta inicialmente con corrección máxima en el ojo derecho (OD) es de 0,7 y en el ojo izquierdo (OI) de 0,6.

El examen funduscópico del OD (fig. 1 A) muestra una lesión macular de coloración amarillenta, redondeada, formando un nivel inferior (lesión viteliforme en fase de pseudohipopion). En el OI (fig. 2 A), se observa una lesión amarillenta subfoveal en estadio viteliforme. La angiografía fluoresceínica (AFG) completa el estudio de la lesión (figs. 1 B y 2 B).

La Tomografía Óptica de Coherencia (OCT) - protocolo de espesor macular- del OD muestra en la zona inferior de la lesión una hiperreflectividad subretiniana con atenuación de la luz a su paso,

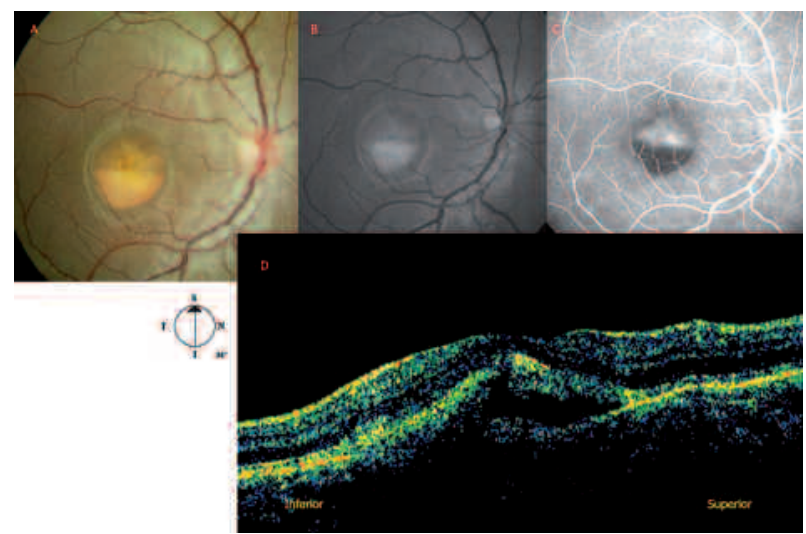

Fig. 1: Ojo derecho: A) Retinografía: muestra una lesión macular en fase de pseudohipopion. B) Retinografía aneritra. C) AFG: hipofluorescencia inferior, por efecto pantalla. D) OCT: hiperreflectividad subretiniana, correspondiente al material depositado e hiporreflectividad correspondiente al líquido subretiniano. correspondiente al material depositado. La zona superior de la lesión presenta una hiporreflectividad correspondiente al líquido subretiniano, que separa el EPR y el neuroepitelio (fig. 1 D). En el ojo izquierdo (fig. 2 D) se observa una sobreelevación de la retina con aumento de reflectividad subretiniana (material depositado y EPR) y atenuación de los tejidos subyacentes. Hay una pérdida de definición de las dos bandas hiperreflectivas observadas en condiciones normales a este nivel.

A los 8 meses (fig. 3), se observa una pérdida severa de la AV, de cuenta dedos a 2 metros con corrección máxima en ambos ojos. En el examen del fondo de ojo presenta un estadio cicatricial en ambos ojos. La OCT muestra una atrofia retiniana asociada a fibrosis subretiniana residual, que se muestra como una disminución del espesor retiniano y una hiperreflectividad subretiniana.

\section{Caso 2}

El padre, de 46 años de edad, refiere haber visto mal desde niño. La agudeza visual con corrección máxima es de 0,1 en el OD y de 0,175 en el OI. En el examen del fondo de ojo muestra una lesión cicatricial en ambos ojos con hiperplasia del EPR asociada. La OCT del OD demuestra una severa atrofia a nivel foveal y en el OI, la existencia de líquido seroso subretiniano y pequeños gránulos hiperreflectivos en el perfil externo de retina neurosensorial, similar a imagen que se observan en coriorretinopatía serosa central crónica (fig. 4).

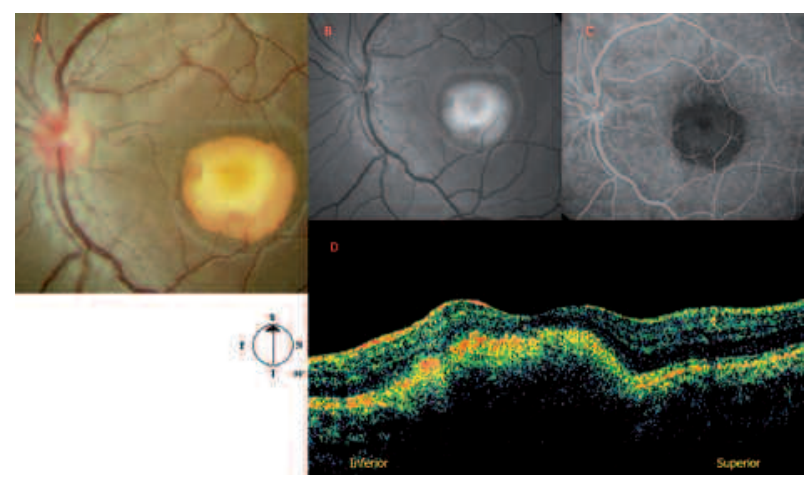

Fig. 2: Ojo izquierdo: A) Retinografía: lesión amarillenta redondeada a nivel subfoveal (estadio viteliforme); B) Retinografía aneritra; C) AFG: muestra bloqueo de la fluorescencia que produce la lesión; D) OCT: sobreelevación de la retina con aumento de reflectividad subretiniana y atenuación de los tejidos subyacentes. 


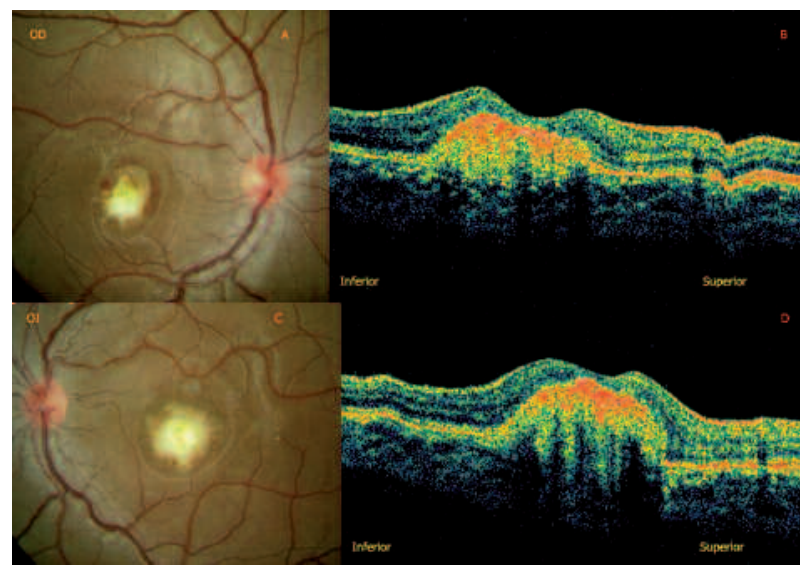

Fig. 3: A y C) Retinografías OD y OI respectivamente, se encuentra estadio cicatricial; $B$ y D) OCT OD y OI respectivamente, muestra aumento de reflectividad subretiniana por componente fibrótico y atrofia coriorretiniana, permitiendo el paso de luz a capas más profundas.

\section{DISCUSIÓN}

A pesar de las características llamativas de la lesión, la VMD 2 mantiene buena agudeza visual inicial. El deterioro de la visión es secundario, a menudo, a la atrofia retiniana suprayacente, o menos frecuentemente a hemorragia, cicatrización o membrana neovascular coroidea.

En esta enfermedad se produce una acumulación de un material amarillento en el centro de la mácula, de forma circunscrita y cupuliforme, en la fase viteliforme, pero hay una cantidad variable de líqui-

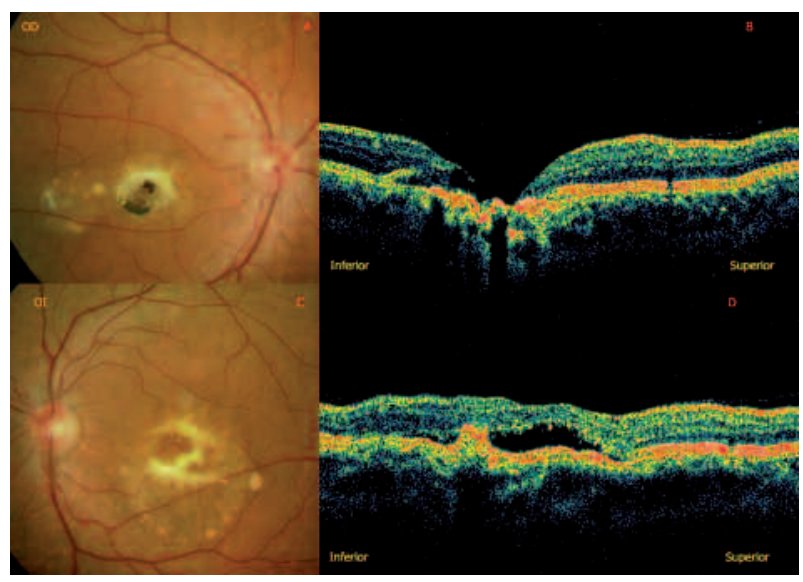

Fig. 4: A) Retinografía OD: cicatriz macular; B) OCT OD: atrofia y pérdida de tejido neurorretiniano foveal; C) Retinografía OI: imagen de etapa vitelorruptiva; $D$ ) OCT OI: desprendimiento seroso y acúmulo de material de alta reflectividad en zona externa de retina sensorial. do subretiniano entremezclado, cuya proporción se incrementa con el tiempo (1). Este material acumulado tiende a ir sedimentándose, formando un nivel entre el mismo y el líquido subretiniano claro. La evolución normal es hacia su desaparición, comenzando desde la parte central de la lesión macular (1) quedando los depósitos en el margen de la lesión.

Las características de las distrofias viteliformes pueden ser evaluadas por OCT, pero hay algunas controversias sobre la localización del material depositado (4). Según estudios histopatológicos, se ha descrito que este material puede encontrarse subEPR o subretiniano (5). El modelo de OCT Stratus ${ }^{\circledR}$ demuestra que el material depositado se localiza entre las 2 capas de alta reflectividad que corresponden al epitelio pigmentario retiniano y la que corresponde a la unión de segmentos externos e internos de los fotorreceptores $(4,5)$. La separación de ambas por el depósito es especialmente visible en los márgenes (4). En nuestro caso, si observamos la imagen del OD (fase de pseudohipopión), en la zona superior de la lesión se muestra la presencia de una hiperreflectividad en el margen correspondiente con la banda del EPR e hiporreflectividad correspondiente con la presencia de líquido subretiniano. En casos más avanzados se puede hacer un seguimiento de la lesión, pues el espesor retiniano suprayacente a la lesión se correlaciona con la agudeza visual.

Se demuestra la utilidad de la Tomografía Óptica de Coherencia como herramienta complementaria que puede aportar datos morfológicos de la lesión, la localización del material depositado, así como el estadio, y si aparecen cambios secundarios en la retina suprayacente.

\section{BIBLIOGRAFÍA}

1. Spaide RF, Noble K, Morgan A, Freund KB. Viteliform macular dystrophy. Ophthalmology 2006; 113: 1392-1400.

2. Rosenblatt BJ, Shah GK. Cystic retinal changes demostrated by optical coherence tomography in best disease. Retina 2004; 24: 322-324.

3. Men G, Batioglu F, Ozkan SS, Atilla H. Ozdamar Y, Aslan $O$. Best's vitelliform macular dystrophy with pseudohypopyon: an optical coherence tomography. Am J Ophtalmol 2004; 137: 963-965.

4. Benhamou N, Messas-Kaplan A, Cohen Y, Gaudric A, Sovied EH, Soubrane G, et al. Adult-onset foveomacular vitelliform dystrophy with OCT 3. Am J Ophthalmol 2004; 138: 294-296.

5. Pianta MJ, Aleman TS, Cideciyan AV, Sunness JS, Li Y, Campochiaro BA, et al. In vivo micropathology of Best macular dystrophy with optical coherence tomography. Exp Eye Res 2003; 76: 203-211. 